\title{
Estimation of Accidental Coincidences in PET
}

\author{
J.F. OLIVER ${ }^{a, *}$ AND M. RAFECAS ${ }^{a, b}$ \\ ${ }^{a}$ Instituto de Física Corpuscular, IFIC (CSIC-UV), C/. Catedrático José Beltrán, 2, E-46980 Paterna, Spain \\ ${ }^{b}$ Werner Siemens Imaging Center, Department for Preclinical Imaging and Radiopharmacy, \\ Eberhard Karls University, Tuebingen, Germany
}

\begin{abstract}
Accidental coincidences are one of the main sources of image degradation in positron emission tomography. It is possible to compensate for their degradation effects, but an accurate method to estimate the randoms rate is required. Two conventional methods are used for random rate estimation: the "singles rate" method and the "delayed window" method. In this work we propose a mathematical model that describes the process of accidental coincidence formation. By using it, we are able to predict the correct number of randoms as well as the estimations provided the singles rate and delayed window methods. The model is used to propose a novel estimation method: the "singles-prompt". The aim of this work is to assess the performance of the singles-prompt method and, specially, the model capabilities at several levels. The results agree with all the predictions of the model. In particular, the singles rate and delayed window estimations behave as described by the model and the singles-prompt method estimates the correct number of randoms regardless of the source distribution and total activities.
\end{abstract}

DOI: 10.12693/APhysPolA.127.1449

PACS: 87.57.uk, 87.57.nf

\section{Introduction}

Accidental coincidences are one of the main sources of image degradation in positron emission tomography (PET) imaging. They reduce image quality and hamper accurate quantitative studies. It is possible to compensate for their degradation effects, but an accurate method to estimate the random rate is then required. Two conventional methods are used for randoms rate estimation: the "singles rate" method (SR) and the "delayed window" method (DW). The SR method offers good statistical properties in the form of low variance, but tends to overestimate the correct value. The DW method estimation is more accurate, although it presents a higher variance.

In this work we propose a mathematical model that accurately describes the process of accidental coincidence formation. The model is able to predict, based on its parameters, the random coincidences between two detectors. By properly taking into account the impact of the rest of the detectors in the scanner, the model is able to describe the pile up effect that takes place at high activities. In addition, it is also able to predict the estimations that will be provided by the SR and DW methods. Moreover, this mathematical model has been used to propose a novel randoms rate estimation model called "singlesprompts" (SP) method.

The model has been validated at several levels. First, synthetic data between two detectors has been generated and used to extract coincidences. The model predictions have been compared with the results. Second, MonteCarlo simulated data of a PET scanner for several phantoms has been used to check the predictions of the model.

*corresponding author; e-mail: oliver@ific.uv.es

\section{The model}

The proposed mathematical model is based on that proposed by [1]. It is based on the next hypotheses: The signal sought in PET is the detection in coincidence of the two photons originated after the $\mathrm{e}^{+}$annihilations. These annihilations follow a Poisson distribution. Once the two photons are created, it may happen: (a) no photon is detected, (b) only one of them is detected (the other is lost), or (c) both are detected (in two different detectors/crystals). Since this is a classification, all the three cases follow a Poisson distribution. Thus, for each pair of detectors we can model the photons as coming from two kinds of sources. The uncorrelated source, describing the case (b), and the correlated source (one for each detector pair) describing the case (c), are shown in Fig. 1.

The model predicts that the randoms rate between two detectors, $i$ and $j$, reads [2]:

$$
\hat{R}_{i j}=2 \tau \lambda_{i} \lambda_{j} \mathrm{e}^{-2 \Lambda \tau},
$$

where $\lambda_{k}$ is the constant associated to the uncorrelated source of detector $k$ and $\Lambda \equiv \sum_{i} \lambda_{i}+1 / 2 \sum_{i, j} \delta_{i j}$. However, $R_{i j}$ cannot be directly computed from data acquisitions since it relies on parameters of the model, $\lambda_{i}, \lambda_{j}$ and $\delta_{i j}$. To overcome this problem, a novel random estimator based on the present model has been proposed [3]: the "singles-prompts" (SP) method that allows obtaining the estimation of Eq. (1) by using the singles and prompts rates

$$
R_{i j}^{\mathrm{SP}} \equiv \frac{2 \tau \mathrm{e}^{-(\lambda+S) \tau}}{(1-2 \lambda \tau)^{2}}\left(S_{i}-\mathrm{e}^{(\lambda+S) \tau} P_{i}\right)\left(S_{j}-\mathrm{e}^{(\lambda+S) \tau} P_{j}\right),
$$

where $S=\sum_{i} S_{i}$ is the rate of singles of the scanner measured as a whole, $P_{i}=\sum_{j} P_{i j}$ is the prompts rate in detector $i$ and $P=\sum_{i} P_{i}$ is twice the prompts rate detected by the scanner. The value of $\lambda$ fulfills $2 \tau \lambda^{2}-\lambda+S-P \mathrm{e}^{(\lambda+S) \tau}=0$. SP requires the same 


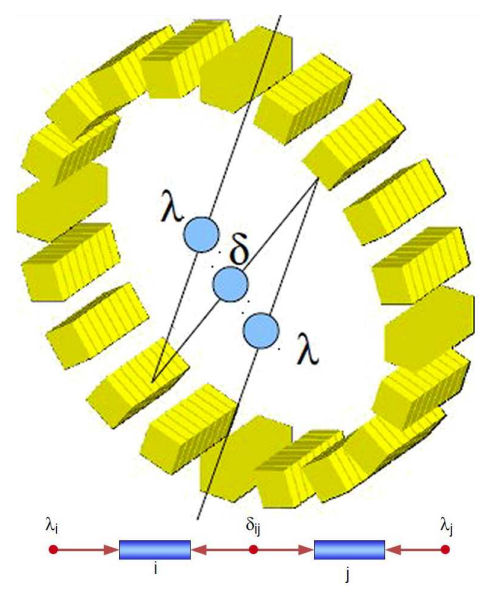

Fig. 1. Top: the test scanner used in this work. The bubbles represent positron annihilations and the lines represent the trajectory of the resulting annihilation photons. Bottom: schematic representation of the model.

measurements that SR and its mathematical complexity is the same since it can be expressed as

$$
R_{i j}^{\mathrm{SP}}=2 \bar{\tau} \bar{S}_{i} \bar{S}_{j},
$$

where the effective time coincidence window, $\bar{\tau}$, and the effective singles count rates $\bar{S}_{i}$ are given by $\bar{\tau}=$ $\tau \mathrm{e}^{-\tau(\lambda+S)} /(1-2 \tau \lambda)^{2}$ and $\bar{S}_{k}=S_{k}-P_{k} \mathrm{e}^{\tau(\lambda+S)}$.

Moreover, the model is also able to predict the outcome of the different estimation methods (not only the correct randoms rate):

$$
\begin{aligned}
& \hat{R}_{i j}^{\mathrm{SR}}=2 \tau\left(\lambda_{i}+\delta_{i}\right)\left(\lambda_{j}+\delta_{j}\right), \\
& \hat{R}_{i j}^{\mathrm{DW}}=\tau\left[\lambda_{i}\left(\lambda_{j}+\delta_{j} / 2\right)+\lambda_{j}\left(\lambda_{i}+\delta_{i} / 2\right)\right] \mathrm{e}^{-2(\lambda+\delta / 2) \tau} .
\end{aligned}
$$

Thus the estimation of the total randoms rate in the scanner, $\hat{R}$, as well as the predictions of the methods may be estimated

$$
\begin{aligned}
& \hat{R}=\tau \lambda^{2} \mathrm{e}^{-2(\lambda+\delta / 2) \tau} \\
& \hat{R}^{\mathrm{SR}}=\tau(\lambda+\delta)^{2}, \hat{R}^{\mathrm{SR}} / \hat{R}=(1+\delta / \lambda)^{2} \mathrm{e}^{2(\lambda+\delta / 2) \tau} \\
& \hat{R}^{\mathrm{DW}}=\tau \lambda(\lambda+\delta / 2) \mathrm{e}^{-2(\lambda+\delta / 2) \tau}, \hat{R}^{\mathrm{DW}} / \hat{R}=(1+\delta / 2 \lambda),(8) \\
& \hat{R}^{\mathrm{SP}}=\tau \lambda^{2} \mathrm{e}^{-2(\lambda+\delta / 2) \tau}, \hat{R} \mathrm{SP} / \hat{R}=1
\end{aligned}
$$

Note that, in general, the model predicts: $\hat{R}^{\mathrm{SR}} \geq \hat{R}^{\mathrm{DW}} \geq$ $\hat{R} \mathrm{SP}=\hat{R}$. Since $\delta$ and $\lambda$ are proportional to the total activity, the equations predict a constant overestimation for DW regardless of the activity. A similar prediction stems for SR when the activity is low enough such that the exponential can be approximated by one. For high activities, an exponential disagreement is expected for SR. Moreover, in the absence of correlated signal, $\delta=0$, the model predicts $\hat{R}^{\mathrm{SR}} \mathrm{e}^{-2 \lambda \tau}=\hat{R}^{\mathrm{DW}}=\hat{R} \mathrm{SP}=\hat{R}$. The DW provides the correct estimation as well as SR. For the latter the activity needs to be low to approximate the exponential by one, i.e. neglecting pile-up.

\section{Model assessment}

\subsection{Synthetic data}

A first validation of the model has been done by using synthetic data. For this part of the study, the system considered was only two individual detectors. Two values for the uncorrelated sources intensities, $\lambda_{1}$ and $\lambda_{2}$, were selected randomly together with a third value for the correlated source intensity, $\delta_{12}$. The timestamps of the detections were created by generating samples extracted from the Poisson distributions described by the intensities. Then, coincidences were extracted. Finally, the total number of randoms was computed, $R$, together with the $\mathrm{SR}$ and DW estimations, $R^{\mathrm{SR}}$ and $R^{\mathrm{DW}}$. The predictions of the model based on the selected intensities were also computed. Several combinations of intensities were generated.

\subsection{Monte-Carlo simulations}

A small animal PET scanner consisting of 8 axial rings of 20 modules each was implemented, Fig. 1. Each module contains a $4 \times 1$ matrix of $2 \times 2 \times 8 \mathrm{~mm}^{3}$ LSO crystals read out individually. The inner diameter is $80 \mathrm{~mm}$ and its axial length is $19.5 \mathrm{~mm}$. An energy resolution of $15 \%$ at $511 \mathrm{keV}$ and a time resolution of $10 \mathrm{~ns}$ were implemented. The energy windows used was [400 keV, $750 \mathrm{keV}]$. The output data format was set to singles list-mode. To investigate dependences on the source distribution, we implemented several phantoms. They range from extended to point-like sources. Specifically, the phantoms are:

- Point-like. A dimensionless phantom in which all the activity is concentrated in a point.

- Mouse-like. To simulate a source distribution with the approximated extent of a mouse we have implemented a homogeneously active cylinder of diameter $35 \mathrm{~mm}$ and height $70 \mathrm{~mm}$.

- Rat-like. Similarly, to approximate a rat we have implemented a homogeneously active cylinder of diameter $70 \mathrm{~mm}$ and height $140 \mathrm{~mm}$.

- Disc. A homogeneously active cylinder of diameter $70 \mathrm{~mm}$ and $10 \mathrm{~mm}$ height. It has been placed outside the scanner, at $70 \mathrm{~mm}$ of the center of the scanner with its symmetry axis coincident with the scanner axis. This phantom will not produce correlated events, i.e. $\delta=0$.

\section{Results}

\subsection{Synthetic data}

For all the combinations generated, the model was able to accurately predict the outcomes. A small sample of tested configurations is shown in Table I, where all the rates are shown in units of $\tau$. 
TABLE I

Synthetic data tested configurations.

\begin{tabular}{l|l|l|l|l|l|l}
\hline \hline$\left\{\lambda_{1}, \lambda_{2}, \delta_{12}\right\}$ & $R$ & $\hat{R}\left(=R^{\mathrm{SP}}\right)$ & $R^{\mathrm{SR}}$ & $\hat{R}^{\mathrm{SR}}$ & $R^{\mathrm{DW}}$ & $\hat{R}^{\mathrm{DW}}$ \\
\hline$\{0.01,0.01,0.0\}$ & 0.000191 & 0.000192 & 0.000200 & 0.0002 & 0.000194 & 0.000192 \\
$\{0.0,0.0,0.01\}$ & 0.0 & 0.0 & 0.0002 & 0.0002 & 0.0 & 0.0 \\
$\{0.01,0.01,0.01\}$ & 0.000188 & 0.000188 & 0.000799 & 0.0008 & 0.000282 & 0.000283 \\
$\{0.0275,0.0150,0.0382\}$ & 0.000704 & 0.000700 & 0.00700 & 0.00698 & 0.00140 & 0.00139 \\
$\{0.0969,0.178,0.0809\}$ & 0.0178 & 0.0170 & 0.0921 & 0.0921 & 0.0236 & 0.0224 \\
$\{0.0104,0.0110,0.0180\}$ & 0.000212 & 0.000212 & 0.00165 & 0.00165 & 0.000392 & 0.000390 \\
$\{0.0273,0.0522,0.0539\}$ & 0.00220 & 0.00218 & 0.0172 & 0.0172 & 0.00386 & 0.00382 \\
$\{0.0171,0.0113,0.0224\}$ & 0.000350 & 0.000350 & 0.00267 & 0.00267 & 0.000637 & 0.000638
\end{tabular}

TABLE II

Simulated data tested configurations.

\begin{tabular}{l|l|l|l|l|l|l|l|l|l|l|l|l}
\hline \hline \multirow{2}{*}{$\mathrm{A}(\mathrm{mCi})$} & \multicolumn{4}{|c|}{ Rat } & \multicolumn{5}{c|}{ Mouse } & \multicolumn{4}{c}{ Point } \\
\cline { 2 - 12 } & $R$ & $R^{\mathrm{SP}}$ & $R^{\mathrm{SR}}$ & $\hat{R}^{\mathrm{SR}}$ & $R$ & $R^{\mathrm{SP}}$ & $R^{\mathrm{SR}}$ & $\hat{R}^{\mathrm{SR}}$ & $R$ & $R^{\mathrm{SP}}$ & $R^{\mathrm{SR}}$ & $\hat{R}^{\mathrm{SR}}$ \\
\hline 0.01 & 2.15 & 2.13 & 2.22 & 2.22 & 3.94 & 3.92 & 4.07 & 4.08 & 4.04 & 3.97 & 5.46 & 5.47 \\
0.1 & 213 & 213 & 222 & 223 & 396 & 397 & 409 & 410 & 402 & 395 & 546 & 547 \\
1 & 22600 & 22600 & 24200 & 24200 & 40600 & 40600 & 44400 & 44400 & 40700 & 39900 & 59200 & 59300
\end{tabular}

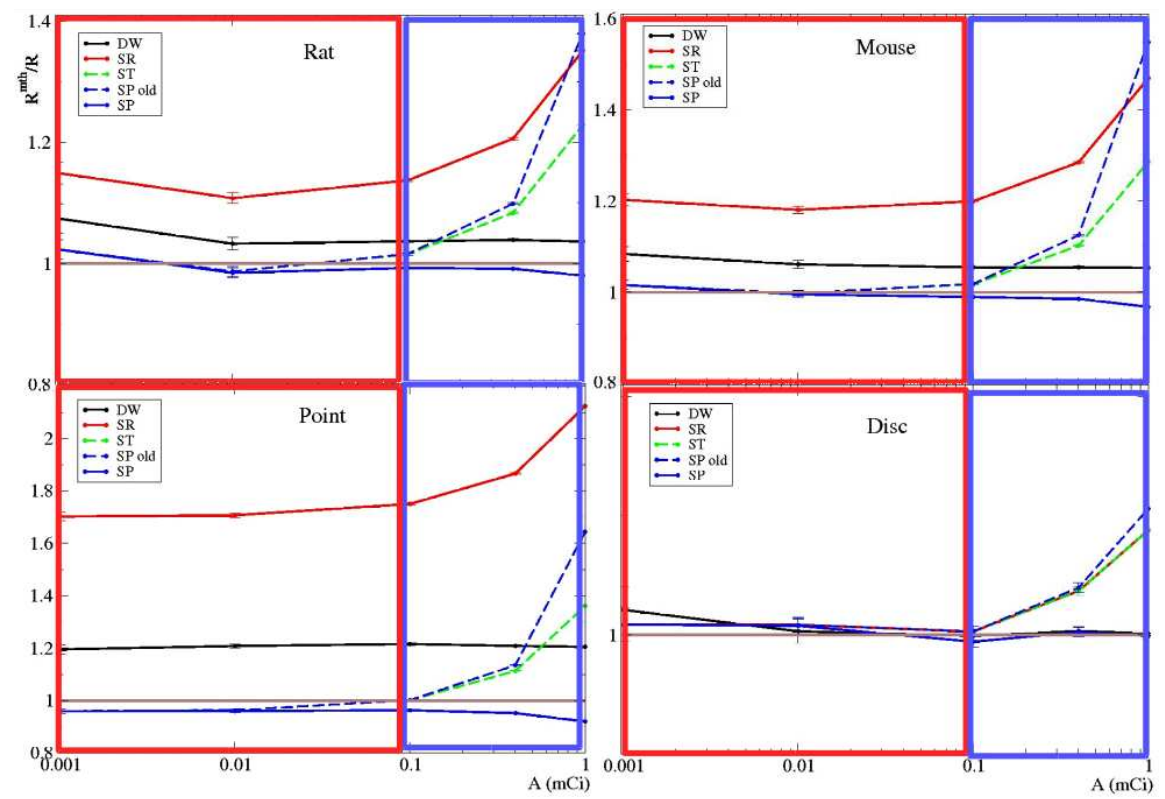

Fig. 2. Solid blue line corresponds to SP, red to SR and black to DW.

\subsection{Simulated data}

The results of the simulated data are shown in Table II and in Fig. 2.

\section{Discussion}

The results obtained with the synthetic data support the validity of the model which is able to predict, for each case, the correct number of randoms as well as the estimations that SR and DW offer.

The results obtained with the (more realistic) simulated data further support the validity of the model. Again, the correct randoms rate is predicted as well as the SR estimation (DW was not checked at this stage). The estimations of the methods can be seen in Fig. 2 . The graphs suggest two different regimes: above and 
below $0.1 \mathrm{mCi}$. For low activities, below $0.1 \mathrm{mCi}$, the pile up can be ignored. For this case, the model predicts a constant overestimation for DW and SR than can be seen in the graphs (left part). Moreover, the results are in agreement with the predicted overestimations, i.e. higher for SR than for DW. For high activities, the model predicts an exponential deviation for SR, see Eq. (7), that clearly appears in the graphs (right part). For DW, the disagreement is predicted to be the same for all activities, see Ref. (8). It is worth to stress that the model predicts that SP provides the correct estimation for all activities and geometries what, actually, agrees with the results.

The case of the disc is very enlightening since it provides a realistic scenario without correlated data, i.e. $\delta=0$. For low activities, all the methods are able to provide the correct randoms estimations as deduced in Sect. 2, while for high activities, the SR overestimation increases exponentially as expected.

\section{Conclusions}

In this work we have presented a model of the process of the accidental coincidences formation which allowed us to propose a new randoms rate estimation method, $R_{i j}^{\mathrm{SP}}=2 \bar{\tau} \bar{S}_{i} \bar{S}_{j}$. The SP estimation has the same structure as the $\mathrm{SR}$ and requires exactly the same measurements. In addition, the model is not only able to predict the correct randoms rate, but also to correctly estimate the outcomes of the SR and DW methods. The model offers a solid framework to understand the behaviour of the SR and DW in different scenarios, i.e. with different source geometries, activities, angular coverages, etc. The SP estimation that is extracted from the model agrees with the correct randoms rate within $1 \%$ (one sigma). Studies to be presented elsewhere show enhancements in conventional FoMs used for image quality assessment when the SP is used in the randoms compensation process.

\section{References}

[1] D.F. Yu, A. Fessler, Nucl. Instrum. Methods Phys. Res. A 488, 362 (2002).

[2] J.F. Oliver, M. Rafecas, Phys. Med. Biol. 55, 6951 (2010).

[3] J.F. Oliver, M. Rafecas, IEEE Nucl. Sci. Symp. Med. Imag. Conf. Rec. 2012, Ed. Bo Yu, IEEE, Anaheim 2012, p. 2295. 